\title{
Dupuytren Contracture Recurrence Project: Reaching Consensus on a Definition of Recurrence
}

\author{
Morbus Dupuytren: Internationale Konsensus Konferenz zur Definition des \\ Rezidivs
}

Authors

Affiliations
N. Felici ${ }^{1}$, I. Marcoccio ${ }^{2}$, R. Giunta ${ }^{3}$, M. Haerle ${ }^{4}$, C. Leclercq ${ }^{5}$, G. Pajardi ${ }^{6}$, S. Wilbrand ${ }^{7}$, A. V. Georgescu ${ }^{8}$, G. Pess

Affiliation addresses are listed at the end of the article

\author{
Key words \\ dupuytren \\ - recurrence \\ - finger flexion \\ contracture \\ Schlüsselwörter \\ - Morbus Dupuytren \\ - Rezidiv \\ - Fingerbeugung \\ - Kontraktur
}

received $\quad 1.10 .2014$

accepted $\quad 6.10 .2014$

\section{Bibliography}

DOI http://dx.doi.org/ 10.1055/s-0034-1394420

Online-Publikation: 20.11.2014

Handchir Mikrochir Plast Chir

2014; 46: 350-354

(c) Georg Thieme Verlag KG

Stuttgart · New York

ISSN 0722-1819

\section{Correspondence}

\section{Ignazio Marcoccio, MD}

Centro di Chirurgia della Mano e Microchirurgia Ortopedica Istituto Clinico Città di Brescia Via Gualla 15

25123 Brescia

Italy

info@ignaziomarcoccio.it

\section{Abstract}

$\nabla$

The aim of this study was to determine a definition of recurrence of Dupuytren disease that could be utilized for the comparison of the results independently from the treatment used. 24 hand surgeons from 17 countries met in an international consensus conference. The participants used the Delphi method to evaluate a series of statements: (1) the need for defining recurrence, (2) the concept of recurrence applied to the Tubiana staging system, (3) the concept of recurrence applied to each single treated joint, and (4) the concept of recurrence applied to the finger ray. For each item, the possible answer was given on a scale of 1-5: 1=maximum disagreement; 2 =disagreement; 3 =agreement; 4 =strong agreement; 5 =absolute agreement. There was consensus on disagreement if 1 and 2 comprised at least $66 \%$ of the recorded answers and consensus on agreement if 3, 4 and 5 comprised at least $66 \%$ of the recorded answers. If a threshold of $66 \%$ was not reached, the related statement was considered "not defined". A need for a definition of recurrence was established. The presence of nodules or cords without finger contracture was not considered an indication of recurrence. The Tubiana staging system was considered inappropriate for reporting recurrence. Recurrence was best determined by the measurement of a specific joint, rather than a total ray. Time 0 occurred between 6 weeks and 3 months. Recurrence was defined as a PED of more than $20^{\circ}$ for at least one of treated joint, in the presence of a palpable cord, compared to the result obtained at time 0 . This study determined the need for a standard definition of recurrence and reached consensus on that definition, which we should become the standard for the reporting of recurrence. If utilized in subsequent publications, this will allow surgeons to compare different techniques and make is easier to help patients make an informed choice.

\section{Zusammenfassung \\ $\nabla$}

Ziel der Konsensuskonferenz in Rom war es, eine Definition des Rezidivs bei Morbus Dupuytren festzulegen, die für einen Vergleich der Ergebnisse unabhängig von der gewählten Behandlung einsetzbar ist. 24 Handchirurgen aus 17 Ländern kamen im Rahmen einer internationalen Konsensuskonferenz zusammen. Die Teilnehmer wendeten die Delphi-Methode an, um folgende Thesen zu bewerten: (1) die Notwendigkeit einer Definition des Rezidivs bei Morbus Dupuytren, das Rezidiv soll (2) auf die Stadieneinteilung nach Tubiana, (3) für jedes einzelne behandelte Gelenk, oder (4) auf den Fingerstrahl angewandt werden. Jede These konnte auf einer Skala von 1 bis 5 bewertet werden: 1 =stimme gar nicht $\mathrm{zu} ; 2$ =stimme nicht zu; 3 =stimme zu; 4 =stimme sehr zu; 5 =stimme vollständig zu. Keine Zustimmung lag vor, wenn mindestens 66\% der erfassten Antworten auf 1 und 2 entfielen und Zustimmung lag vor, falls mindestens $66 \%$ der erfassten Antworten auf 3, 4 und 5 entfielen. Falls der Schwellenwert von 66\% nicht erreicht wurde, wurde die jeweilige Aussage als „nicht definiert“ betrachtet.

Die Notwendigkeit einer Definition des Rezidivs bei Morbus Dupuytren wurde mit dieser Methode festgestellt. Das Vorliegen von Knoten oder Sehnen ohne Kontraktur der Finger wurde nicht als Anzeichen eines Rezidivs gewertet. Die Stadieneinteilung nach Tubiana stellte sich als ungeeignet zur Beschreibung eines Rezidivs heraus. Ein Rezidiv bei Morbus Dupuytren ist besser durch die Messung eines spezifischen Gelenks als durch eine Messung der Beugekontraktur des gesamten Fingerstrahls bestimmt.

Der Ausgangspunkt Null wurde definiert als Zeitraum zwischen 6 Wochen und 3 Monaten nach der primären Behandlung. Ein Rezidiv wird dann definiert als eine passive Beugekontraktur von mehr als $20^{\circ}$ für jedes behandelte Gelenk bei Vorliegen eines tastbaren Stranges, verglichen mit dem Ergebnis zum Zeitpunkt Null. 
Die Konferenz zeigte die Notwendigkeit einer Definition des Rezidivs bei Morbus Dupuytren und führte zu einem Konsens in Bezug auf diese Definition. Bei Verwendung in nachfolgenden Publikationen wird sie es ermöglichen, verschiedene Techniken zu vergleichen und die Patienten beim Treffen einer informierten Entscheidung zu unterstützen.

\section{Introduction}

$\nabla$

Dupuytren disease (DD) is a well-known, slowly progressive fibroproliferative disorder that affects the hand. The very first medical description dates back to 1614, when Felix Plater of Basel reported in his Observationum the progression of ulnar finger contracture. In 1777, Henry Cline and, later in 1882, his disciple, Astley Cooper, described the role of palmar aponeurosis and performed a procedure similar to the modern mini-invasive open fasciotomy. However, it was Baron Guillaume Dupuytren, chief surgeon at the Hôtel-Dieu in Paris, who described the permanent contracture of the fingers on 5th December 1831 and then published his observation in 1832 in the Leçon Orales de Clinique Chirurgicale [1].

Despite the time elapsed, currently there is no consensus as to which is the gold standard for the treatment of DD, even if surgery is the most common option. There are a variety of alternatives for treatment, including dermofasciectomy, standard limited fasciectomy, segmental fasciectomy, radical fasciectomy, open fasciotomy, percutaneous needle fasciotomy/needle aponeurotomy [2-4] and the injection of collagenase Clostridium histolyticum (CCH) [5]. At the moment no outcome studies can absolutely sustain the superiority of one method over another and this is mainly due to the lack of standardization of the patient cohorts and the absence of a clear definition of recurrence [6]. The published recurrence rates are extremely variable, with a range from 2 to $86 \%$ [7-9]. It seems that the selection of the best treatment choice largely depends on surgeon's experience and confidence with one technique, rather than being based on the patient's need, or degree and extension of the disease, or objective and scientific superiority of one technique over another. A clear definition of recurrence and a standard way to report recurrence would allow physicians to compare treatments and help patients make informed decisions [10]. The recurrence rate thus is a key factor not only in clinical settings when considering treatment options, but influences economy in the health-care system and modifies medico-legal and insurer perspectives [2,3].

In order to allow a clear comparison of the results of specific techniques, a clear definition of recurrence in DD is mandatory. A group of international hand surgeons formed a consensus conference, utilizing the Delphi method, to establish that definition.

\section{Materials and Methods \\ $\nabla$}

The Delphi method is an iterative process to collect and distill the anonymous judgments of experts using a series of data collection and analysis techniques interspersed with feedback. The Delphi method is well suited as a research instrument when there is incomplete knowledge about a problem or phenomenon. The original Delphi method was developed in the 1950s by Norman Dalkey, of the RAND Corporation, for a US-sponsored military project [11]. This method is primarily based on the ano- nymity of participants, which allows the participants to freely express their opinions without any social pressure to conform to others in the group. Decisions are evaluated on their merit, rather than on who has proposed the idea; on iteration, which allows participants to refine their views; on controlled feedback, informing the participants of the other participant's perspectives, which provides the opportunity to clarify or change views; and on statistical aggregation of group responses, which allows for quantitative analysis and data interpretation [12].

The Delphi method was used by the present authors to reach a consensus on the definition of recurrence in DD.

Based on an idea of the first author, members from the Italian Society for Surgery of the Hand (SICM) and the Federation of European Societies for Surgery of the Hand (FESSH) gathered to form the Scientific Committee (SC) of the Dupuytren Contracture Recurrence Project (DCRP). The SC developed the research statements, which were divided into 4 groups: (1) the need for defining recurrence, (2) the concept of recurrence applied to the Tubiana staging system, (3) the concept of recurrence applied to each single treated joint, and (4) the concept of recurrence applied to the finger ray. For each item, the possible answer was given on a scale of $1-5: 1=$ maximum disagreement; 2 = disagreement; 3 =agreement; 4 =strong agreement; 5=absolute agreement. There was consensus on disagreement if 1 and 2 comprised at least $66 \%$ of the recorded answers and consensus on agreement if 3,4 and 5 comprised at least $66 \%$ of the recorded answers. If a threshold of $66 \%$ was not reached, the related statement was considered "not solved" in terms of consensus ( $\bullet$ Table $\mathbf{1}$ ).

The SC selected members from the SICM, FESSH and IFSSH based on their clinical experience, reputation and prior publications on the recurrence of DD. 62 surgeons were identified and took part to the DCRP. Round 1 of the Delphi method was an on-line questionnaire and was completed by 44 of them from 17 different countries. The consensus conference was held in Rome on April 22, 2013, to discuss the results of the Delphi questionnaire and reach an ultimate and shared definition of DD recurrence. All the participants of the on-line survey were invited to Rome, 24 hand surgeons accepted the invitation to participate. 3 additional rounds of the Delphi method were necessary to reach the consensus on the definition of recurrence ( $\bullet$ Table 2 ).

\section{Results}

The first group of statements was the need for defining recurrence. The group confirmed the need to find a clear and detailed definition of recurrence in DD that could be applied for any length of follow-up.

The second group of statements was the concept of recurrence applied to the Tubiana staging system (Tss). The Tss utilizes an algebraic sum of the degree of contracture of all the treated joints of a specific finger ray. It was not considered to be an accurate tool for assessing recurrence. There was also consensus that the presence of a new hand nodule could not be considered a recurrence. 
Table 1 Research statements - Delphi round 1

\section{Statement 1. Need for defining recurrence}

1.1 I think that a clear and agreed definition of the concept of recurrence in Dupuytren's disease can be useful.

1.2 In the literature, there is a definition of Dupuytren's disease recurrence which is widely used by hand surgeons to compare the results obtained with different treatments.

1.3 The ideal definition of Dupuytren's disease recurrence should apply to the clinical examination of the hand regardless of the treatment used. In this way, it would be possible to compare the results obtained with different techniques (fasciectomy, fasciotomy, collagenase, etc.) by using the same measurement.

1.4 There is no need to define the concept of Dupuytren's disease recurrence.

1.5 The definition of recurrence should be independent from the duration of follow-up: it should be possible to apply it with the same efficacy after a month or after several months from treatment.

\section{Statement 2. Concept of recurrence applied to the Tubiana staging system}

2.1 The new occurrence of simple hand nodules can be considered as a recurrence.

2.2 The occurrence of a flexion retraction causing an extension deficit to one of the finger rays treated should be considered as a recurrence.

2.3 The return to the pre-treatment Tubiana stage can be defined as a recurrence.

2.4 An increase of 1 Tubiana grade as compared to the result obtained with the treatment can be considered as recurrence.

2.5 In patients with PIPs involvement, recurrences are more common. This concept should be considered when we try to find a definition of recurrence aimed at assessing exactly what is the treatment efficacy; therefore, the increase of 1 Tubiana stage as compared to the result obtained with the treatment in patients who had grade 1 or 2 at baseline and the increase of 2 Tubiana stages in patients with stage 3 or 4 at baseline can be considered as a recurrence.

2.6 The occurrence of a flexion retraction on a finger ray different from those treated should be considered as a recurrence.

2.7 The flexion retraction of the same finger ray previously treated should be considered as a recurrence.

2.8 In the Tubiana staging system, the algebraic sum of the PED (passive extension deficit) of the different treated joint is used and therefore it is not an accurate tool for assessing the occurrence of a recurrence.

\section{Statement 3. Concept of recurrence applied to each single treated joint}

3.1 In order to obtain an accurate and specific measure, it is useful to measure the PED (passive extension deficit) of each treated joint showing a flexion retraction and the TPED (total passive extension deficit) of each finger ray before and immediately after the treatment.

3.2 When the flexion retraction affects the same joint as previously treated, it can be defined as a recurrence.

3.3 When the retraction takes the single treated joint back to the same pre-treatment extension deficit, it can be defined as a recurrence.

3.4 A recurrence should involve any PED (passive extension deficit) increase of one of the treated joint.

3.5 The occurrence of an extension deficit of $>15^{\circ}$ for an MP as compared to the extension obtained with the treatment and the occurrence of an extension deficit of $>20^{\circ}$ for a PIP as compared to the extension obtained with the treatment can be defined as a recurrence.

3.6 The increase of $>30 \%$ of the delta between pre-op PED (passive extension deficit) and post-op PED of each single treated joint can be defined as a recurrence: if a treated joint has a pre-treatment PED of $50^{\circ}$ and it decreases to $0^{\circ}$ after treatment, a recurrence is defined as the occurrence of a flexion retraction with a PED of at least $15^{\circ}$.

3.7 An increase of $>30 \%$ of the delta between pre-op PED (passive extension deficit) and post-op PED for MPs and $>50 \%$ for PIPs can be considered as a recurrence.

Statement 4. Concept of recurrence applied to the finger rays

4.1 The concept of recurrence should be applied to the single finger ray and not to each single treated joint.

4.2 A recurrence should involve an increase of $>30 \%$ of the delta between pre-op TPED (total passive extension deficit) and post-op TPED of each finger ray treated: if the TPED of a finger is $100^{\circ}$ before treatment and is decreased to $0^{\circ}$ after treatment, a recurrence is defined as the occurrence of a flexion retraction with a TPED $>30^{\circ}$.

4.3 A recurrence should involve an increase of $>90 \%$ of the delta between pre-op TPED (total passive extension deficit) and post-op TPED of each finger ray treated: if the pre-treatment TPED of a finger is $100^{\circ}$ and is reduced to $0^{\circ}$ after treatment, a recurrence is defined as the occurrence of a new flexion retraction with a TPED $>90^{\circ}$.

4.4 A recurrence should involve any increase of the delta between pre-op TPED (total passive extension deficit) and post-op TPED.

The third group of statements was the concept of recurrence applied to each single treated joint. The group determined that to assess an extension deficit, the measurement of the passive extension deficit (PED) of each treated joint should be used.

The fourth group of statements was the concept of recurrence applied to the finger ray. Measuring the total passive extension deficit (TPED) of the entire finger ray was discussed. No consensus was reached at the first round of Delphi. A second round defined an agreement in favor of the individual PED measurement of each treated joint, considering each joint as a separate entity.

The definition of time 0 , the time when treatment results can be considered stable and follow-up measurements performed, was also considered. It took 3 rounds of Delphi to reach consensus. Time 0 was defined as the period between 6 weeks and 3 months after treatment.

At this point, the group agreed that a definition of recurrence in DD was necessary. It was decided which was the best way to measure recurrence (individual joint PED) and when time 0 was present post-treatment ( 6 weeks -3 months). Finally, a standard degree of recurrence was needed for reporting purposes. Through clear consensus, a PED of more than $20^{\circ}$ for at least one of treated joints, in the presence of a palpable cord, compared to the result obtained at time 0 represented the definition of recurrence.

\section{Discussion}

$\nabla$

Dupuytren disease is a slowly progressive fibroproliferative disease of unknown origin for which there is no medical cure. The historical definitions of recurrence, which include those of Heuston "the smallest palpable nodule in an operated field" [13], Gordon "the disease in the same area" [14], and Tubiana "the reappearance of Dupuytren's contracture tissue in a zone previously operated" [15], have been followed by many new definitions [2$4,7-10,16]$. In the report of Kan et al. [8], only $49 \%$ of the 113 articles studied gave a definition of recurrence. Among those, $63 \%$ 
Table 2 Research statements - Delphi rounds 2 and 3.

Delphi round 2

Statement 2. Concept of recurrence applied to the Tubiana staging system

2.1 The new occurrence of simple hand nodules can be considered as a recurrence.

2.2 In patients with PIPs involvement, recurrences are more common. This concept should be considered when we try to find a definition of recurrence aimed at assessing exactly what is the treatment efficacy; therefore, the increase of 1 Tubiana stage as compared to the result obtained with the treatment in patients who had grade 1 or 2 at baseline and the increase of 2 Tubiana stages in patients with stage 3 or 4 at baseline can be considered as a recurrence.

Statement 4. Concept of recurrence applied to the finger rays

4.1 The concept of recurrence should be applied to the single finger ray and not to each single treated joint.

4.2 A recurrence should involve an increase of $>30 \%$ of the delta between pre-op TPED (total passive extension deficit) and post-op TPED of each finger ray treated: if the TPED of a finger is $100^{\circ}$ before treatment and is decreased to $0^{\circ}$ after treatment, a recurrence is defined as the occurrence of a flexion retraction with a TPED $>30^{\circ}$.

4.3 A recurrence should involve an increase of $>90 \%$ of the delta between pre-op TPED (total passive extension deficit) and post-op TPED of each finger ray treated: if the pretreatment TPED of a finger is $100^{\circ}$ and is reduced to $0^{\circ}$ after treatment, a recurrence is defined as the occurrence of a new flexion retraction with a TPED $>90^{\circ}$.

4.4 A recurrence should involve any increase of the delta between pre-op TPED (total passive extension deficit) and post-op TPED.

Statement 5. How to evaluate recurrence

5.1 In order to assess the occurrence of a recurrence a functional evaluation should be preferred to a histopathological study.

5.2 The occurrence of a finger contracture is an expression of a recurrence only in the presence of a palpable cord.

5.3 Time 0 : the point where treatment results can be considered stable.

-1 pre-operative

-26 weeks post-operative

-33 months post-operative

- 46 months post-operative

5.4

The degree of contracture of each single treated joint considered as recurrence at Time 0 :

-1 any

$-220^{\circ}$

$-330^{\circ}$

-4 Other

Delphi round 3

6.1 The point where treatment results can be considered stable is defined as TIME 0 and it is between 6 weeks and 3 months.

of recurrences were based on the return of nodules or cords in the operated hand, $27 \%$ were based on the return of contraction with an angular threshold that varied from 1 to $50^{\circ}$, and $10 \%$ were based on the patient's self-report. He concluded that an international consensus on the definition of recurrence is needed to allow the comparison of recurrence rates and thus treatments.

Werker et al. [9], in a selection of 21 reports, found that the definition of recurrence was qualitative in $95 \%$ of the studies, including the appearance of new Dupuytren's tissue within the area cleared at operation, the reappearance of Dupuytren's disease in the cleared operative field, and the appearance of new fascial bands in an area where fasciectomy had been previously performed. That study indicated the importance of a quantitative definition of recurrence, as the possibility of comparing the long-term efficacy of different treatments for Dupuytren's contracture is dependent upon such a definition. To predict the chance of recurrence, Rombouts et al. [16] proposed a histological staging of DD into 3 types: proliferative, fibrocellular and fibrous. Statistical analysis indicated a significant relationship between histological classification and the recurrence rate, which was higher in the proliferative type and lower in the fibrous type. An agreement on the definition of recurrence would allow the differentiation of true recurrence from extension of the disease [16].

The first group of statements reached consensus on the need to define recurrence. The significance of the presence of a new nodule in the operated field was discussed. The occurrence of a simple nodule can only be considered as an indication of recurrence when radical surgery is performed (fasciectomy). It is not an indication of recurrence after fasciotomy, needle aponeurot- omy or $\mathrm{CCH}$ injection. When evaluating those treatments, the definition of recurrence based on the return of contracture measured in angular degrees is more appropriate. Since the aim of this study was to find a definition of recurrence that could be applied following any treatment procedure available, the presence of nodules or cords without finger contracture cannot be considered as an indication of recurrence. Excluding any qualitative methods or an anatomic description to define recurrence, only quantitative and functional measurements remain available.

The Tss is a measurement of the whole digital ray. The mathematical sum of the degrees of contracture of the metacarpalphalangeal (MP) joint, proximal interphalangeal (PIP) joint and distal interphalangeal (DIP) joint of the affected finger ray are classified into 4 stages of increasing severity: $0-45^{\circ}, 45-90^{\circ}$, $90-135^{\circ}$ and $>135^{\circ}$. In Tss, each stage is separated from the other by $45^{\circ}$, which represents a wide spectrum. Another problem arises when the post-operative evaluation is made, since it is impossible to extract from the Tubiana stage the exact degree of involvement of a single affected joint. Comparison of the longterm efficacy of different treatments for DD is only possible if a quantitative definition of results referring to an individual treated joint was applied to the measurement of outcomes during the follow-up period. As a result of the Delphi questionnaire, the Tss was considered an inappropriate tool to assess recurrence in DD and goniometric measurement of the single treated joint expressed as angular degrees of PED was preferred.

When measurements of recurrence are made in terms of PED, the keystone parameter is the threshold angular degree over which recurrence is defined. In fact, the $45^{\circ}$ range of each stage in the Tss and the $1-50^{\circ}$ range in threshold variability illustrated 
in other reports are able to alter the recurrence rate up to $20 \%$ [8]. In the current literature, the choice of angular threshold is variable, even though it represents the critical parameter. The minimum angular threshold should not be less than $5-10^{\circ}$, which represents the measurement error of a goniometer [17]. In our study, we analyzed different angular degree thresholds for each single treated joint (any; $20^{\circ} ; 30^{\circ}$ and others), and consensus was reached defining recurrence at greater than $20^{\circ}$.

To make comparisons of results homogeneous, it is important to determine the time after treatment when measurements should begin. This is the point where treatment results can be considered stable. After 3 rounds of the Delphi method, participants in the consensus conference reached a consensus on the definition of time 0 , which was set as the period between 6 weeks and 3 months after treatment.

The development of new methods for the treatment of DD has broadened the options for hand surgeons. Unfortunately, there are no clear guidelines that allow the results of these treatments to be compared. There is also no method available to determine which of the different treatments should be utilized, according to the degree and stage of the disease. In the present study, the authors have tried to develop a standard definition of recurrence that could be utilized in future papers and presentations. This will help surgeons compare different techniques and be able to help patients make an informed choice. With this study the authors aimed to find a definition that can be applied to all the currently available methods of treatment, both surgical and non-surgical.

The definition that emerged from the consensus conference does not take into consideration patients' perspective and the definition has not been tested on a patient cohort. Moreover, in the Rome conference (Delphi rounds 2 and 3) 24 surgeons participated in respect of the 44 in round 1 . These elements can be considered as limitations of this report.

The reduced number of participants in the Rome conference in the authors' opinion did not jeopardize the result as $75 \%$ of statements were solved in terms of consensus during the first Delphi round. In order to respect the Delphi procedures during the live conference the voting system was anonymous and electronically managed.

The authors are firmly convinced that the definition of recurrence in DD must be independent from the procedure adopted to treat the disease that is the reason why in the present study we chose not to apply the definition of recurrence on a patient series, leaving this chance to future reports.

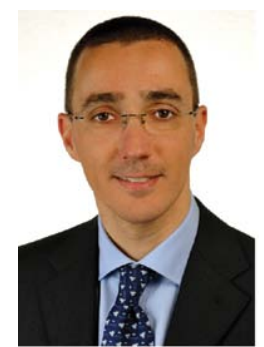

\section{Ignazio Marcoccio}

1998 M.D. cum laude, University of Catania, Italy; 2005 - Specialization in Orthopaedic and Traumatology cum laude University of Modena, Italy; 2007 - Degree in Microsurgical techniques, La Sorbonne - Paris; 2005 - present Assistant at the Hand and Microsurgical centre at the Istituto Clinico Città di Brescia (Gruppo Ospedaliero San Donato), Brescia - Italy; 2009 - present Coordinator and former Member of the scientific commission for the Advanced Italian Microsurgery Course, Naples - Italy; 2008 - present Council member of the Italian Microsurgery and Reconstructive Technique Group (G.I.M.E.T.R.A.); 2002 - present Treasurer and former
Secretary and Council member of the Italian Society of Microsurgery. Member of the Italian Society for Surgery of the Hand, European Wrist Arthroscopy Association, Italian Society for Elbow and Shoulder Surgery.

\section{Conflict of Interest: Rome conference was sponsored by Pfizer.}

Affiliations

${ }^{1}$ Direttore ad interim UOC Chirurgia Plastica e Chirurgie della Mano A.O.R.N. „San Camillo-Forlanini“ Roma - Italy

${ }^{2}$ Instituto Clinico Città di Bressica, Unità Operativa di Chirurgia della Mano e Microchirurgia Ortopedica, Brescia - Italy

${ }^{3}$ Chefarzt der Handchirurgie, Plastische Chirurgie und Ästhetische Chirurgie, Campus Innenstadt und Grosshadern der Ludwig-Maximilians Universität München - Germany

${ }^{4}$ Head of Department, Plastic and Hand Surgery, Orthopaedic Clinic, Markgroeningen(Stuttgart) - Germany

${ }^{5}$ Institut de la Main Clinique Jouvenet, Paris - France

${ }^{6}$ Director Of Hand Surgery Department - St. Joseph Hospital MultiMedica Group Milan - Italy Plastic Surgery School - University of Milan Italy

${ }^{7}$ Senior Consultant Department of Hand Surgery Akademiska University Hospital, Uppsala

${ }_{8}^{8}$ Professor in Plastic Surgery and Reconstructive Microsurgery University of Medicine Iuliu Hatieganu Spitalul Clinic de Recuperare Clinic of Plastic Surgery and Reconstructive Microsurgery

${ }^{9}$ Chief, Central Jersey Hand Surgery, Eatontown, NJ Clinical Senior Instructor Department of Orthopedic Surgery, Drexel University School of Medicine, Philadelphia, PA

\section{References}

1 Tubiana R. History. In: Tubiana R, Leclercq C, Hurst LC, Badalamente MA, Mackin EJ. (eds.) Dupuytren's disease. $1^{\text {st }}$ Edn. London: Martin Dunitz Ltd; 2000; 1-11

2 Peimer CA, Blazar $P$, Coleman $S$ et al. Dupuytren contracture recurrence following treatment with collagenase Clostridium histolyticum (CORDLESS study): 3-year data. J Hand Surg Am 2013; 38: 12-22

3 Peimer CA, Skodny P, Mackowiak JI. Collagenase Clostridium histolyticum for Dupuytren contracture: Pattern of use and effectiveness in clinical practice. J Hand Surg Am 2013; 38: 2370-2376

4 Pess GP, Pess RM, Pess RA. Results of needle aponeurotomy for Dupuytren contracture in over 1,000 fingers. J Hand Surg Am 2012; 37 : 651-656

5 Hurst LC, Badalamente MA, Hentz VR et al. Injectable collagenase Clostridium histlyticum for Dupuytren's contracture. N Engl J Med 2009; 361: 968-979

6 Degreef I, De Smet L. Dupuytren disease: on our way to a cure? Acta Orthop Belg 2013; 79: 243-249

7 Becker GW, Davis TR. The outcome of surgical treatments for primary Dupuytren's disease - a systematic review. J Hand Surg Br 2010; 35: 623-626

8 Kan HJ, Verrijp FW, Huisstede BMA et al. The consequences of different definitions for recurrence of Dupuytren's disease. J Plast Reconstr Aesthet Surg 2013; 66: 95-103

9 Werker PMN, Pess GM, van Rijssen AL et al. Correction of contracture and recurrence rates of Dupuytren contracture following invasive treatment: the importance of clear definitions. J Hand Surg Am 2012; 37: 2095-2105

10 Degreef I, De Smet L. Risk factors in Dupuytren's diathesis: is recurrence after surgery predictable? Acta Orthop Belg 2011; 77: 27-32

11 Dalkey $N$, Helmer $O$. An experimental application of the Delphi method to the use of experts. Manage Sci 1963; 9: 458-467

12 Rowe $G$, Wright $G$. The Delphi technique as a forecasting tool: issues and analysis. Int J Forecasting 1999; 15: 353-375

13 Hueston JT. Recurrent Dupuytren's contracture. Plast Recontr Surg 1963; 31: 66-69

14 Gordon S. Dupuytren's contracture: recurrence and extension following surgical treatment. Br J Plast Surg 1957; 9: 286-288

15 Leclercq $C$. Results of surgical treatment. In: Tubiana R, Leclercq C, Hurst LC, Badalamente MA, Mackin EJ. (Eds.) Dupuytren's disease. $1^{\text {st }}$ Edn. London: Martin Dunitz Ltd; 2000; 239-249

16 Rombouts JJ, Noel H, Legrain $Y$ et al. Prediction of recurrence in the treatment of Dupuytren's disease: evaluation of a histologic classification. J Hand Surg Am 1989; 14: 644-652

17 Ellis $B$, Bruton A. A study to compare the reliability of composite finger flexion with goniometry for measurements of range of motion in the hand. Clin Rehabil 2002; 16: 562-570 